\title{
The Effect of Indium Concentration on the Structure and Properties of Zirconium Based Intermetallics: First-Principles Calculations
}

\author{
Fuda Guo, Junyan Wu, Shuai Liu, and Yongzhong Zhan \\ College of Materials Science and Engineering, Guangxi University, Nanning, Guangxi 530004, China \\ Correspondence should be addressed to Yongzhong Zhan; zyzmatres@aliyun.com
}

Received 14 April 2016; Revised 20 June 2016; Accepted 20 June 2016

Academic Editor: Veer P. S. Awana

Copyright (C) 2016 Fuda Guo et al. This is an open access article distributed under the Creative Commons Attribution License, which permits unrestricted use, distribution, and reproduction in any medium, provided the original work is properly cited.

The phase stability, mechanical, electronic, and thermodynamic properties of In-Zr compounds have been explored using the firstprinciples calculation based on density functional theory (DFT). The calculated formation enthalpies show that these compounds are all thermodynamically stable. Information on electronic structure indicates that they possess metallic characteristics and there is a common hybridization between In-p and $\mathrm{Zr}-\mathrm{d}$ states near the Fermi level. Elastic properties have been taken into consideration. The calculated results on the ratio of the bulk to shear modulus $(B / G)$ validate that $\operatorname{InZr}_{3}$ has the strongest deformation resistance. The increase of indium content results in the breakout of a linear decrease of the bulk modulus and Young's modulus. The calculated theoretical hardness of $\alpha-\mathrm{In}_{3} \mathrm{Zr}$ is higher than the other In-Zr compounds.

\section{Introduction}

Saitovitch et al. made a study on the nuclear probe Cd on In sites of intermetallic compounds of the In-Zr system [1]. However, there is still short of experimental information available on the zirconium-indium system. Experiments on the phase diagram of In-Zr system present the phase compositions of In-Zr compounds [2]. Zumdick et al. examined the crystal structures and properties of $\mathrm{ZrIn}_{2}$ and discussed the chemical bonding of $\mathrm{ZrIn}_{2}$ on the basis of density functional calculations [3]. Meschel and Kleppa studied the standard enthalpies of formation of some transition metal-indium compounds by high-temperature direct synthesis calorimetry and provided some new thermochemical data for $\mathrm{In}-\mathrm{Zr}$ binary systems [4].

To the best of our knowledge, there is no systematically relevant research on the structural stability and mechanical properties of the In- $\mathrm{Zr}$ compounds. Indium possesses unique physical and chemical properties and has been widely applied in the fields of medicine and health, solar battery, national defense and military, aerospace, and modern information industry. The applications of indium compounds and alloys are restricted due to technical level and high cost. Actually, fundamental investigation on indium-related materials is still a challenge.

Recently, advances in computer-aided design and computer-aided manufacturing $(\mathrm{CAD} / \mathrm{CAM})$ technology are used in novel manufacturing routes [5]. First-principles calculation is a reliable and precise approach for studying ground-state properties such as the structure, elastic properties, and phase stability. In this paper, we made a systemic investigation on the structure and properties of zirconium based intermetallics. Structural, mechanical, electronic, and thermodynamic properties of four In-Zr compounds have been analyzed and discussed in detail by performing the firstprinciples calculations. It may improve the knowledge of the In-Zr alloys and provide available theoretical basis for further optimization and design for both indium and zirconium alloys.

\section{Computational Methods}

All calculations were performed by first principle based on density functional theory (DFT) implemented in Cambridge sequential total energy package (CASTEP) code [6]. The 
interaction between ions and electrons was described by using the ultrasoft pseudopotential [7]. The generalized gradient approximation (GGA) with the Perdew-BurkeErnzerhof (PBE) [8] was used for the exchange correlation functional. The electronic configurations in this study were In $4 \mathrm{~d}^{10} 5 \mathrm{~s}^{2} 5 \mathrm{p}^{1}$ and $\mathrm{Zr} 4 \mathrm{~s}^{2} 4 \mathrm{p}^{6} 4 \mathrm{~d}^{2} 5 \mathrm{~s}^{2}$, respectively. We choose various $k$ points here mainly because the different phases possess diverse structure. All parameters including $k$ point convergence test have been carefully tested. To ensure the precision of the calculations, we select firstly the $k$ point and cutoff energy at the fine precision as the initial value and then improve continually precision by change the $k$ point and cutoff energy until the calculated crystal parameters have a great agreement with previous data. The corresponding results were displayed in Table 1 . According to the calculation at the different $k$ points and cutoff energy, the cutoff energy and $k$ point were selected for different phase. In this work, the cutoff energy of $450 \mathrm{eV}$ was set to InZr and $\alpha-\operatorname{In}_{3} \mathrm{Zr}$ and $350 \mathrm{eV}$ was set to $\mathrm{In}_{2} \mathrm{Zr}$. Meanwhile, the cutoff energy of $550 \mathrm{eV}$ was selected for all other phases. Brillouin zone integrations were performed by using a Monkhorst-Pack $k$ point mesh and grids of $12 \times 12 \times 12,7 \times 7 \times 7,6 \times 6 \times 1$, $6 \times 6 \times 2,15 \times 15 \times 10$, and $15 \times 15 \times 8 k$ point were set to be the sampling of $\operatorname{InZr}_{3}, \operatorname{InZr}, \operatorname{In}_{2} \mathrm{Zr}, \alpha-\mathrm{In}_{3} \mathrm{Zr}$, In, and $\alpha$ $\mathrm{Zr}$, respectively. During the structural optimization, all atoms were fully relaxed using conjugate gradient method until the total energy, force, and atomic displacement were less than 1 $\times 10^{-5} \mathrm{eV} / \AA, 0.03 \mathrm{eV} / \AA$, and $0.001 \AA$, respectively.

\section{Results and Discussions}

3.1. Structural Stability. In this section, we established the initial crystal structures of four In-Zr compounds based on the experimental crystallographic data. Then the lattice parameters and internal coordinates of these compounds were optimized with the measure of first-principle calculation. Figure 2 presents the optimized structural models of four In-Zr compounds and Table 2 lists the lattice parameter compared with the available experimental data. The reliability of this work and the reasonability of the computational methodology can be justified from the good agreement between the available experimental data and present work in optimized lattice parameters and atomic coordinates.

The enthalpy of formation was calculated in this part so as to estimate the thermodynamic stability of In-Zr compounds. Enthalpy of formation of $\mathrm{In}-\mathrm{Zr}$ compounds is defined as follows:

$$
\Delta H=\frac{E_{\text {total }}-x E_{\mathrm{In}}^{\mathrm{bulk}}-y E_{\mathrm{Zr}}^{\mathrm{bulk}}}{x+y}
$$

where $E_{\text {total }}$ is the total energy of $\operatorname{In}_{x} \mathrm{Zr}_{y}$ and $E_{\mathrm{In}}^{\text {bulk }}$ and $E_{\mathrm{Zr}}^{\text {bulk }}$ are the total energy of an indium atom and a zirconium atom in the bulk state, respectively. The calculated results are listed in Table 2. The obtained formation enthalpies of the $\mathrm{In}-\mathrm{Zr}$ compounds are all negative, meaning that they are all stable at $0 \mathrm{~K}$ and $0 \mathrm{GPa}[13]$. We can see that the formation enthalpy of InZr is the lowest contrast with $\alpha-\mathrm{In}_{3} \mathrm{Zr}, \mathrm{In}_{2} \mathrm{Zr}$, and $\mathrm{InZr} \mathrm{r}_{3}$,
TABLE 1: The lattice constants ( $\AA$ ) of In-Zr compounds at different $k$ points and cutoff energy.

\begin{tabular}{|c|c|c|c|c|c|}
\hline Phase & $k$ points & Energy cutoff $(\mathrm{eV})$ & $a(\AA)$ & $b(\AA)$ & $c(\AA)$ \\
\hline \multirow{9}{*}{$\mathrm{InZr}_{3}$} & $6 \times 6 \times 6$ & 350 & 4.480 & & \\
\hline & $6 \times 6 \times 6$ & 380 & 4.479 & & \\
\hline & $6 \times 6 \times 6$ & 400 & 4.479 & & \\
\hline & $8 \times 8 \times 8$ & 400 & 4.479 & & \\
\hline & $8 \times 8 \times 8$ & 420 & 4.479 & & \\
\hline & $8 \times 8 \times 8$ & 450 & 4.479 & & \\
\hline & $12 \times 12 \times 12$ & 450 & 4.480 & & \\
\hline & $12 \times 12 \times 12$ & 500 & 4.475 & & \\
\hline & $12 \times 12 \times 12$ & 550 & 4.474 & & \\
\hline \multirow{9}{*}{ InZr } & $6 \times 6 \times 6$ & 350 & 4.473 & & \\
\hline & $6 \times 6 \times 6$ & 380 & 4.472 & & \\
\hline & $6 \times 6 \times 6$ & 400 & 4.471 & & \\
\hline & $7 \times 7 \times 7$ & 400 & 4.469 & & \\
\hline & $7 \times 7 \times 7$ & 420 & 4.470 & & \\
\hline & $7 \times 7 \times 7$ & 450 & 4.470 & & \\
\hline & $8 \times 8 \times 8$ & 400 & 4.471 & & \\
\hline & $8 \times 8 \times 8$ & 420 & 4.471 & & \\
\hline & $8 \times 8 \times 8$ & 450 & 4.471 & & \\
\hline $\mathrm{In}_{2} \mathrm{Zr}$ & $6 \times 6 \times 1$ & 350 & 4.442 & & 27.890 \\
\hline \multirow{6}{*}{$\alpha-\operatorname{In}_{3} \mathrm{Zr}$} & $6 \times 6 \times 1$ & 350 & 4.371 & & 19.514 \\
\hline & $6 \times 6 \times 1$ & 400 & 4.358 & & 19.349 \\
\hline & $6 \times 6 \times 1$ & 450 & 4.364 & & 19.318 \\
\hline & $6 \times 6 \times 2$ & 350 & 4.370 & & 19.514 \\
\hline & $6 \times 6 \times 2$ & 400 & 4.359 & & 19.339 \\
\hline & $6 \times 6 \times 2$ & 450 & 4.364 & & 19.293 \\
\hline
\end{tabular}

which implies that InZr is the most stable among four Zr-In compounds.

3.2. Mechanical Properties. Before considering the mechanical properties, we prefer to study the mechanical stability of these In-Zr compounds. According to the structural feature (see Figure 1), the compounds discussed in this paper mainly consist of two crystal structures such as cubic structure (i.e., $\mathrm{InZr}_{3}$ and $\mathrm{InZr}$ ) and tetragonal structure (i.e., $\mathrm{In}_{2} \mathrm{Zr}$ and $\alpha$ $\mathrm{In}_{3} \mathrm{Zr}$ ). For the cubic crystal, there are three independent elastic constants, that is, $C_{11}, C_{12}$, and $C_{44}$. Counterpart criterion of mechanical stability is based on the following formula [14]:

$$
\begin{aligned}
C_{11} & >0 \\
C_{44} & >0 \\
C_{11} & >\left|C_{12}\right| ; \\
\left(C_{11}+2 C_{12}\right) & >0
\end{aligned}
$$

For tetragonal system, there are six independent elastic constants, that is, $C_{11}, C_{12}, C_{13}, C_{33}, C_{44}$, and $C_{66}$. The 


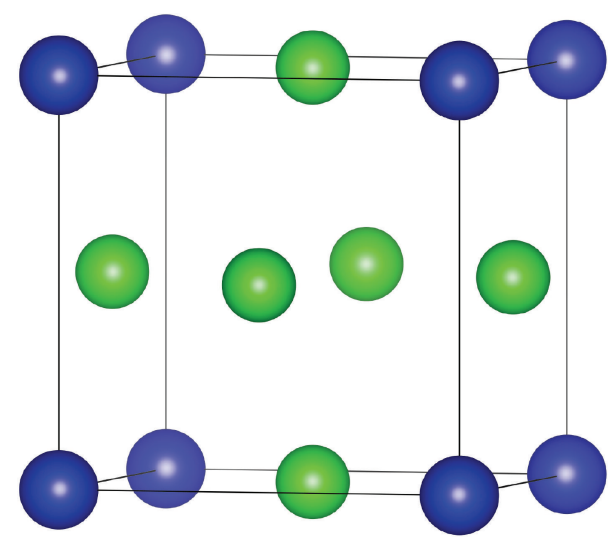

(a) $\mathrm{InZr}_{3}$

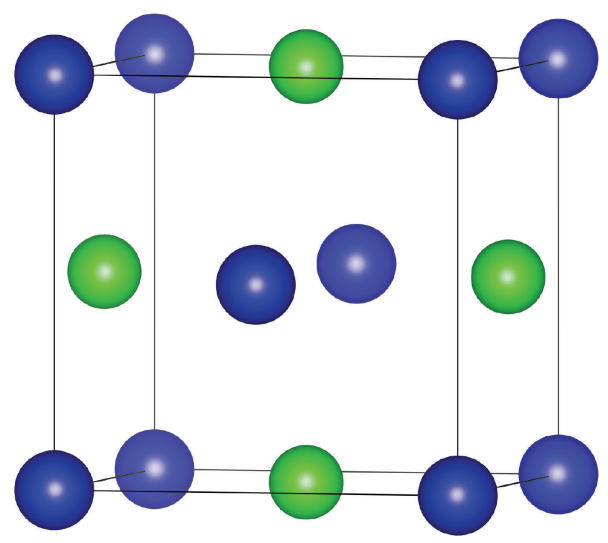

(b) InZr

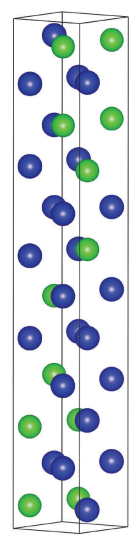

(c) $\mathrm{In}_{2} \mathrm{Zr}$
000 $00 \bullet 00$ $\infty \circ \infty$ $\infty \circ \infty$ - 00 ० 00000 - 000

(d) $\alpha-\mathrm{In}_{3} \mathrm{Zr}$

Figure 1: Structure model of In-Zr compounds. (a) $\operatorname{InZr}_{3}$, (b) InZr, (c) $\mathrm{In}_{2} \mathrm{Zr}$, and (d) $\alpha$-In $\mathrm{I}_{3} \mathrm{Zr}$. Blue and green spheres represent In and $\mathrm{Zr}$ atoms, respectively.

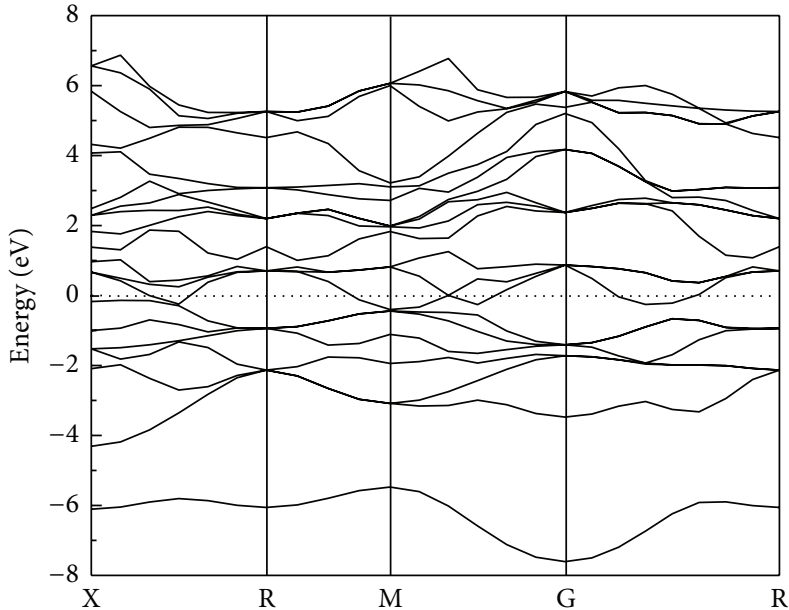

(a) $\mathrm{InZr}_{3}$

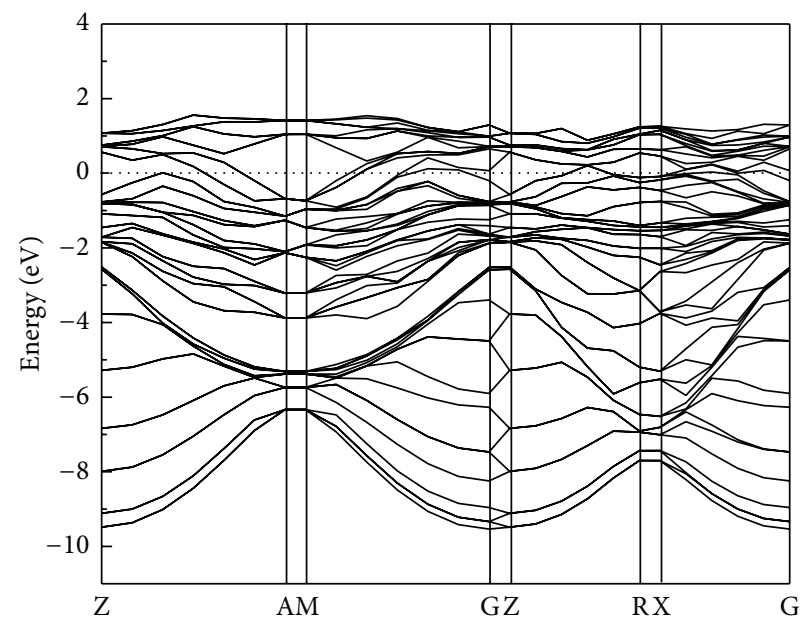

(c) $\operatorname{In}_{2} \mathrm{Zr}$

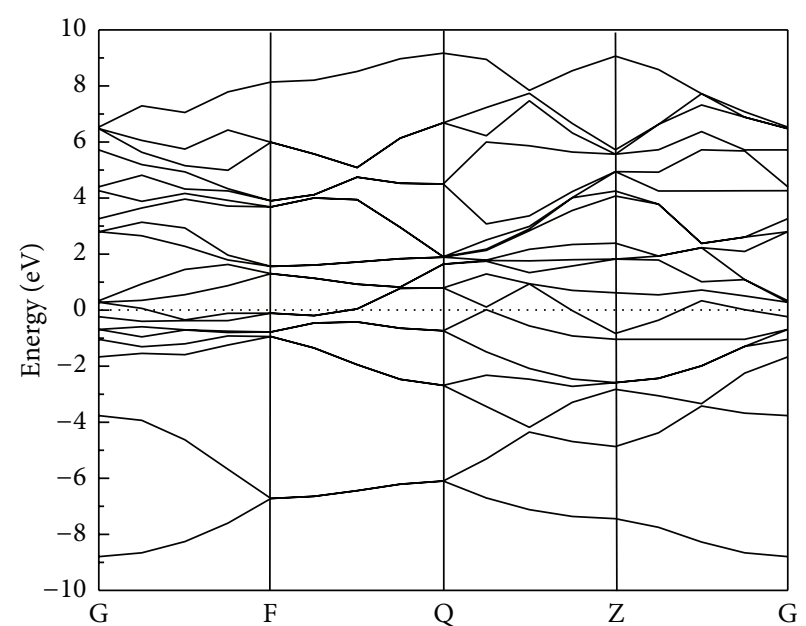

(b) InZr

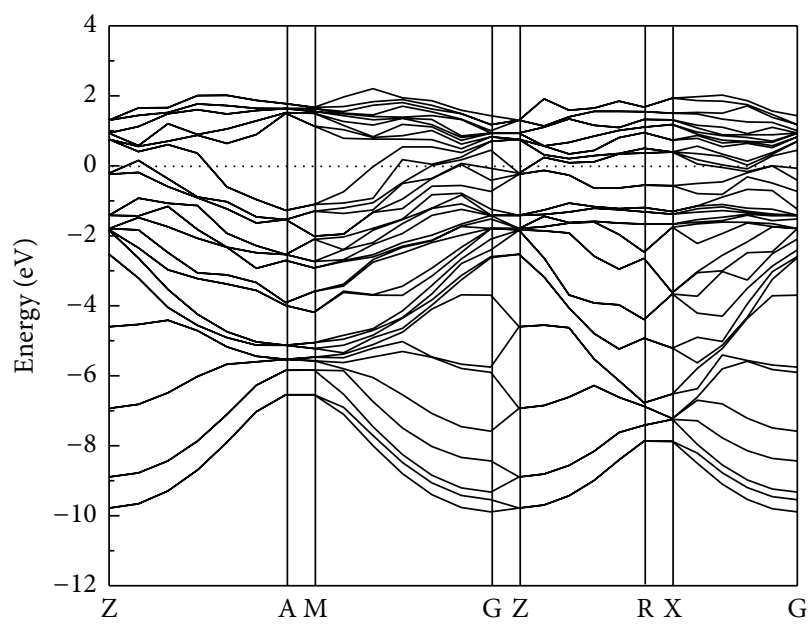

(d) $\alpha-\operatorname{In}_{3} \mathrm{Zr}$

FIgURE 2: The electronic energy band structures near Fermi level of In-Zr intermetallics. The dotted lines denote the Fermi level $E_{F}$. 
TABLE 2: Calculated lattice parameters $(\AA)$ and formation enthalpies (eV/atom) for In, $\alpha$-Zr, and In-Zr intermetallic compounds in comparison with the available experimental data.

\begin{tabular}{|c|c|c|c|c|c|c|c|}
\hline Phase & Space (number) & Prototype & Pearson's symbol & Atomic numbers & $a$ & $b$ & Formation enthalpy (eV/atom) \\
\hline \multirow{4}{*}{$\operatorname{InZr}_{3}$} & \multirow{4}{*}{ Pm-3m (221) } & \multirow{4}{*}{$\mathrm{AuCu}_{3}$} & \multirow{4}{*}{$\mathrm{cP} 4$} & \multirow{4}{*}{4} & 4.474 & & \multirow{4}{*}{-0.247} \\
\hline & & & & & $4.450[2]$ & & \\
\hline & & & & & $4.461[9]$ & & \\
\hline & & & & & $4.460[10]$ & & \\
\hline \multirow{2}{*}{ InZr } & \multirow{2}{*}{ Fm-3m (225) } & \multirow{2}{*}{$\mathrm{Cu}$} & \multirow{2}{*}{$\mathrm{cF} 4$} & \multirow{2}{*}{4} & 4.47 & & \multirow{2}{*}{-0.313} \\
\hline & & & & & $4.418[2]$ & & \\
\hline \multirow{3}{*}{$\mathrm{In}_{2} \mathrm{Zr}$} & \multirow{3}{*}{ I41/amdZ (141) } & \multirow{3}{*}{$\mathrm{HfGa}_{2}$} & \multirow{3}{*}{$\mathrm{tI} 24$} & \multirow{3}{*}{24} & 4.442 & 27.89 & \multirow{3}{*}{-0.284} \\
\hline & & & & & $4.385[2]$ & 27.23 & \\
\hline & & & & & $4.387[3]$ & 27.238 & \\
\hline \multirow{2}{*}{$\alpha-\operatorname{In}_{3} \mathrm{Zr}$} & \multirow{2}{*}{ I4/mmm (139) } & \multirow{2}{*}{$\mathrm{Al}_{3} \mathrm{Zr}$} & \multirow{2}{*}{ tI16 } & \multirow{2}{*}{16} & 4.364 & 19.293 & \multirow{2}{*}{-0.212} \\
\hline & & & & & $4.303[2]$ & 18.94 & \\
\hline \multirow{3}{*}{ In } & \multirow{3}{*}{$\mathrm{I} 4 / \mathrm{mmm}(139)$} & \multirow{3}{*}{ In } & \multirow{3}{*}{$\mathrm{tI} 2$} & \multirow{3}{*}{2} & 3.271 & 5.172 & \\
\hline & & & & & $3.251[2]$ & 4.947 & \\
\hline & & & & & $3.251[11]$ & 4.947 & \\
\hline \multirow{3}{*}{$\alpha-\mathrm{Zr}$} & \multirow{3}{*}{$\mathrm{P} 63 / \mathrm{mmc}(194)$} & \multirow{3}{*}{$\mathrm{Mg}$} & \multirow{3}{*}{ hP2 } & \multirow{3}{*}{2} & 3.23 & 5.175 & \\
\hline & & & & & $3.232[12]$ & 5.148 & \\
\hline & & & & & $3.232[2]$ & 5.148 & \\
\hline
\end{tabular}

qualification for stability can be measured in the following expression [14]:

$$
\begin{aligned}
C_{11} & >0 ; \\
C_{33} & >0 ; \\
C_{44} & >0 ; \\
C_{66} & >0 ; \\
C_{11}-C_{12} & >0 ; \\
C_{11}+C_{33}-2 C_{13} & >0 \\
2\left(C_{11}+C_{12}\right)+C_{33}+4 C_{13} & >0
\end{aligned}
$$

Elastic constants reflect the resistance of a crystal to an applied external stress, whose values will provide valuable information about structural stability [15]. Based on the calculation results, all the In- $\mathrm{Zr}$ compounds conform to the corresponding standard of stability. That is, the In- $\mathrm{Zr}$ compounds considered in this paper are all mechanically stable. The mechanical prospects for all compounds show that four $\mathrm{Zr}$-In intermetallic compounds have stable structure [16].

For researching the mechanical properties of the In- $\mathrm{Zr}$ compounds, the following computational formulas are used [17]:

$$
\begin{aligned}
& B=\frac{1}{2}\left(B_{V}+B_{R}\right), \\
& G=\frac{1}{2}\left(G_{V}+G_{R}\right), \\
& E=\frac{9 G B}{3 B+G} .
\end{aligned}
$$

For cubic system [17],

$$
\begin{aligned}
& B_{V}=B_{R}=\frac{1}{3}\left(C_{11}+2 C_{12}\right) \\
& G_{V}=\frac{1}{5}\left(C_{11}-C_{12}+3 C_{44}\right) \\
& G_{R}=\frac{5\left(C_{11}-C_{12}\right) C_{44}}{3\left(C_{11}-C_{12}\right)+4 C_{44}}
\end{aligned}
$$

Particularly, the InZr compound breaks the symmetry of the cubic system, and the general elastic constants are [18]

$$
\begin{aligned}
& \overline{C_{11}}=\frac{C_{11}+C_{22}+C_{33}}{3}, \\
& \overline{C_{12}}=\frac{C_{12}+C_{13}+C_{23}}{3}, \\
& \overline{C_{44}}=\frac{C_{44}+C_{55}+C_{66}}{3} .
\end{aligned}
$$

For tetragonal system [14],

$$
\begin{aligned}
B_{V} & =\frac{2\left(C_{11}+C_{22}\right)+C_{33}+4 C_{13}}{9}, \\
G_{V} & =\frac{M+3 C_{11}-3 C_{12}+12 C_{44}+6 C_{66}}{30}, \\
B_{R} & =\frac{C^{2}}{M}, \\
G_{R} & =\frac{15}{\left[18 B_{V} / C^{2}+6 /\left(C_{11}-C_{12}\right)+6 / C_{44}+3 / C_{66}\right]}, \\
M & =C_{11}+C_{12}+2 C_{33}-4 C_{13}, \\
C^{2} & =\left(C_{11}+C_{12}\right) C_{33}-2 C_{13}^{2} .
\end{aligned}
$$


From Table 4, it can be seen that $\operatorname{InZr}_{3}$, with the bulk modulus of $97.112 \mathrm{GPa}$, has the strongest resistance to volume change by applied pressure [19] and the strongest average bond strength of atoms for the given crystal [20]. On the contrary, $\alpha-\mathrm{In}_{3} \mathrm{Zr}$ is the weakest one compared with the other In-Zr compounds. As shown in Table 4 the relation between bulk modulus and indium content is monotonically decreasing, which is equivalent to the resistance to volume deformation of the four compounds and the abrupt degradation of average bond strength of atoms with increasing indium content. The shape change capacity of material is usually reflected in the value of shear modulus [15]. The difference of this value between $\operatorname{InZr}_{3}$ and $\alpha$ - $\mathrm{In}_{3} \mathrm{Zr}$ is quite small. The lowest shear modulus is $47.007 \mathrm{GPa}$ for InZr and the highest shear modulus is only $51.6 \mathrm{GPa}$ for $\mathrm{InZr}_{3}$. There is no obvious rule about shear modulus with increasing indium content. What is more, it can be seen that $B>G$ exists in the In-Zr system, indicating that shear modulus limits the mechanical stability of the In- $\mathrm{Zr}$ compounds [21]. Materials with high Young's modulus depict strong resistance to uniaxial tension [22] and indicate the elastic stiffness as well. It can be seen from Table 4 that calculated Young' modulus of $\mathrm{InZr}_{3}$ is $131.509 \mathrm{GPa}$, which is higher than that of the other In- $\mathrm{Zr}$ compounds. Young's modulus of $\alpha-\mathrm{In}_{3} \mathrm{Zr}$ is only about $118.350 \mathrm{GPa}$, which is lower than all the others. We can conclude from above analysis that $E$ has similar change compared with $B$. Young's modulus decreases linearly with increasing indium content. In addition, it should be noted that $C_{12}$ and $C_{44}$ are much lower than $C_{11}$ for all compounds, which implies that all the crystals are more difficult to deform in the axial direction, compared with shear direction [23].

$B / G$ ratio, in general, was applied to describe the brittleness and toughness of materials [24]. The brittle-ductile transition of materials can be judged by the $B / G$ ratio. If the $B / G$ ratio exceeds 1.75 , the material is ductile. Otherwise, it is brittle. From Table 4 , the $B / G$ value of $\alpha-\operatorname{In}_{3} \mathrm{Zr}$ is less than 1.75 , which implies the brittleness of this compound. The $B / G$ values of $\operatorname{InZr}_{3}, I n Z r$, and $\operatorname{In}_{2} Z r$ are all higher than 1.75 , demonstrating that these In-Zr intermetallic compounds are ductile. Poisson's ratio $v$ can be used to forecast the ductility of crystals, as well [25]. From Table 4 , it is shown that $B / G$ and $v$ have similar variation trend with increasing $\mathrm{Zr}$ content. It is found that when $B / G$ is higher than 1.75 , the values of $v$ are more than 0.26 for $\mathrm{InZr}_{3}$, InZr, and $\mathrm{In}_{2} \mathrm{Zr}$. This is consistent with the conclusion that ductile compounds usually have high $v$.

The hardness parameter $H$ can be calculated [26]:

$$
\begin{aligned}
H & =\frac{(1-2 v) E}{6(1+v)}, \\
v & =\frac{3 B-2 G}{2(3 B+G)} .
\end{aligned}
$$

The calculated theoretical hardness was shown in Table 4 . We can know that there is a transition at 0.67 indium content. When indium content is less than 0.67 , the hardness may decrease with increasing indium content. While it is higher than 0.67 , hardness exhibits a big jump and increases with the
TABLE 3: Calculated elastic constants $C_{i j}(\mathrm{GPa})$ for In-Zr compounds.

\begin{tabular}{lcccccc}
\hline Phase & $C_{11}$ & $C_{12}$ & $C_{13}$ & $C_{33}$ & $C_{44}$ & $C_{66}$ \\
\hline $\mathrm{InZr}_{3}$ & 140.589 & 75.373 & & & 70.192 & \\
$\mathrm{InZr}$ & 129.626 & 74.343 & & & 67.083 & \\
$\mathrm{In}_{2} \mathrm{Zr}$ & 124.925 & 63.411 & 49.866 & 224.796 & 42.994 & 56.707 \\
$\alpha-\mathrm{In}_{3} \mathrm{Zr}$ & 129.757 & 53.044 & 42.007 & 172.837 & 40.61 & 63.057 \\
\hline
\end{tabular}

TABLE 4: The calculated shear modulus $G(\mathrm{GPa})$, bulk modulus $B$ $(\mathrm{GPa})$, Young's modulus $E$ ( $\mathrm{GPa})$, bulk modulus to shear modulus ratio $(B / G)$, Poisson's ratio, and Hardness $(\mathrm{GPa})$ as well as available experimental and theoretical data.

\begin{tabular}{lcccccc}
\hline Phase & $B$ & $G$ & $E$ & $H$ & $v$ & $B / G$ \\
\hline $\mathrm{InZr}_{3}$ & 97.112 & 51.6 & 131.509 & 7.764 & 0.274 & 1.882 \\
$\mathrm{InZr}$ & 92.771 & 47.007 & 120.644 & 6.792 & 0.283 & 1.974 \\
$\mathrm{In}_{2} \mathrm{Zr}$ & 87.104 & 47.184 & 119.903 & 15.728 & 0.271 & 1.846 \\
$\alpha-\mathrm{In}_{3} \mathrm{Zr}$ & 78.18 & 47.427 & 118.35 & 15.809 & 0.248 & 1.648 \\
\hline
\end{tabular}

indium increasing. The calculated theoretical hardness of $\alpha$ $\mathrm{In}_{3} \mathrm{Zr}$ is 15.809 , which indicates that $\alpha-\mathrm{In}_{3} \mathrm{Zr}$ is expected to be the hardest among all the In-Zr binary compounds.

3.3. Electronic Properties. In order to study the electronic properties of the In-Zr intermetallic compounds, we calculate the electronic energy band structures and the results are depicted in Figure 2, where the dot dash line zero-point energy signifies the highest energy level occupied by the valence electrons at $0 \mathrm{~K}$. From Figure 2, we can know that all compounds show metallic nature due to the fact that these bands cross $E_{F}$. Meanwhile, the density of states corresponding to the Fermi surface was all positive, which also implies that all these In-Zr intermetallics are conductive phases.

To further understand the electronic structure and mechanical properties of four In-Zr compounds, density of states was calculated and analyzed in detail. Figure 3 represents the total and partial density of states of four In- $\mathrm{Zr}$ compounds, where the black vertical dashed of DOS indicates the Fermi level $\left(E_{F}\right)$. As is vividly demonstrated in Figure 3, the valence electron near $E_{F}$ mainly derived from In-p and $\mathrm{Zr}$-d states. It indicates that the $5 \mathrm{p}$ states of In hybridize strongly with the $\mathrm{Zr} 4 \mathrm{~d}$ states and the In- $\mathrm{Zr}$ atomic bonds formed along the $\mathrm{p}$-d directions near the region Fermi level. Particularly, the DOS profile of $\mathrm{InZr}_{3}$ presents an obvious pseudogap. The pseudogap indicates that the hybridization between In and $\mathrm{Zr}$ induces a separation of the bonding states and antibonding states [13]. That is why $\mathrm{InZr}_{3}$ own the greatest deformation resistance [27].

The distance between the zero energy symbolized bands and the nearest peaks may manifest the bonds transformation of compounds $[28,29]$. In general, the longer the distance to the zero energy symbolized bands, the stronger the covalent bonds. Moreover, with the falling in metallicity of bond, alloy ductility usually decreases [30]. As the DOS shown in Figure 3, the distance adjacent zero energy is the lowest 

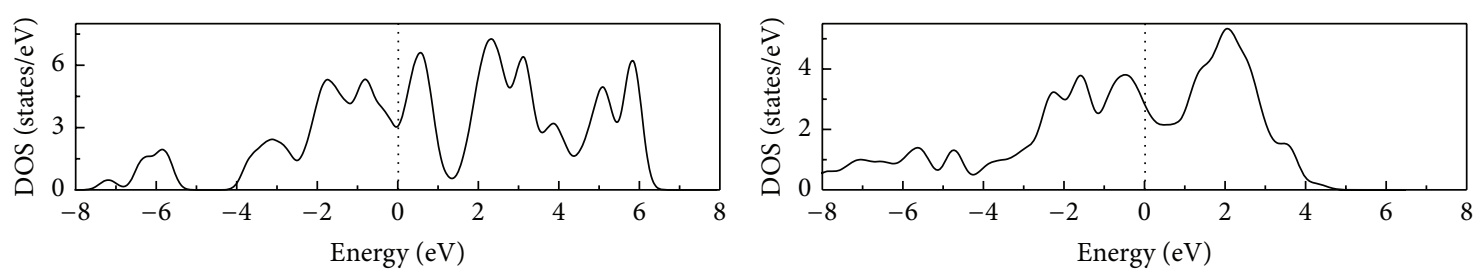

- TDOS

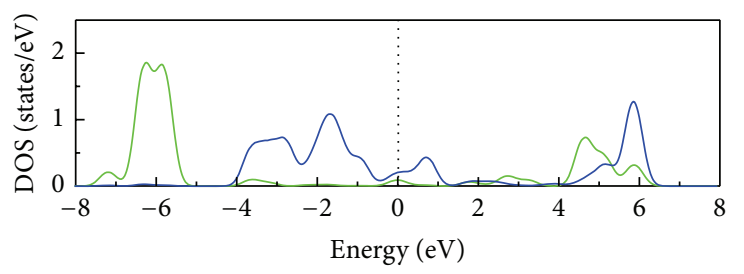

TDOS

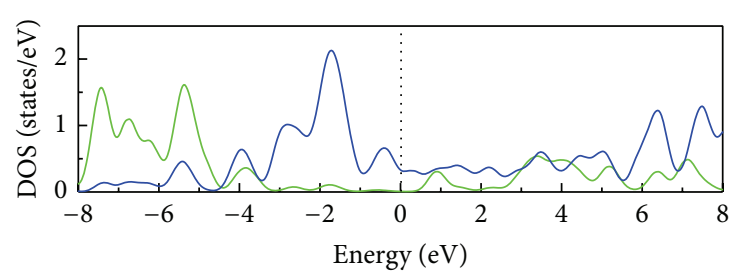

_ In-s

— In-s

— In-p
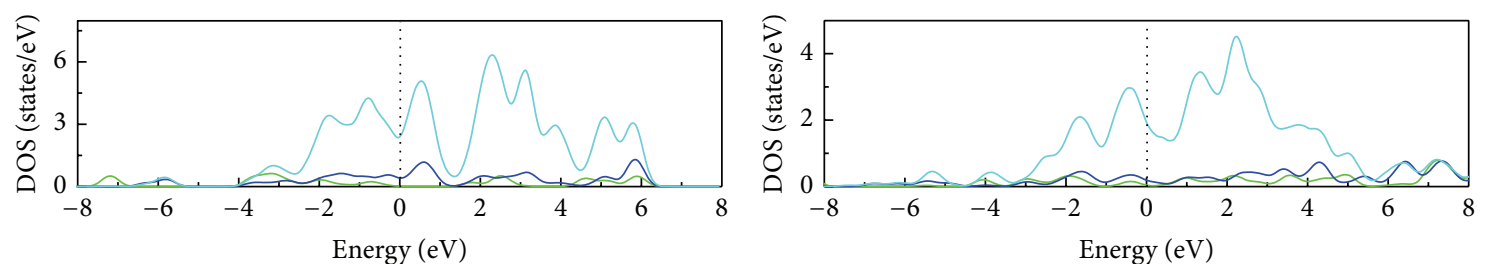

Z Zr-s

Z Zr-d

- Zr-s

Zr-d

(a) $\mathrm{InZr}_{3}$

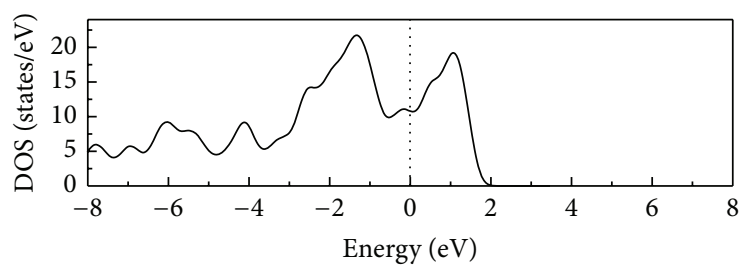

(b) InZr
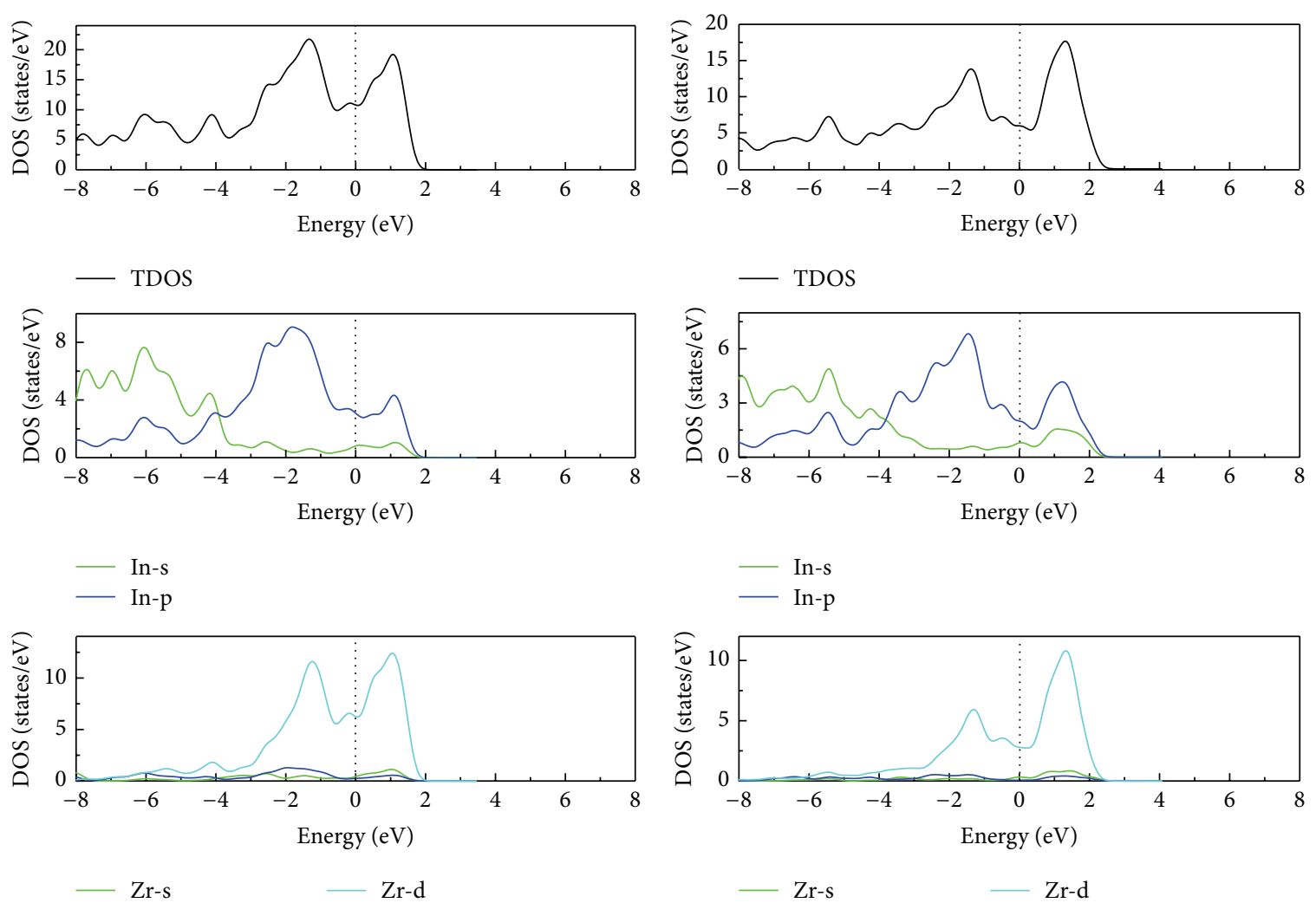

Zr-s
- Zr-p

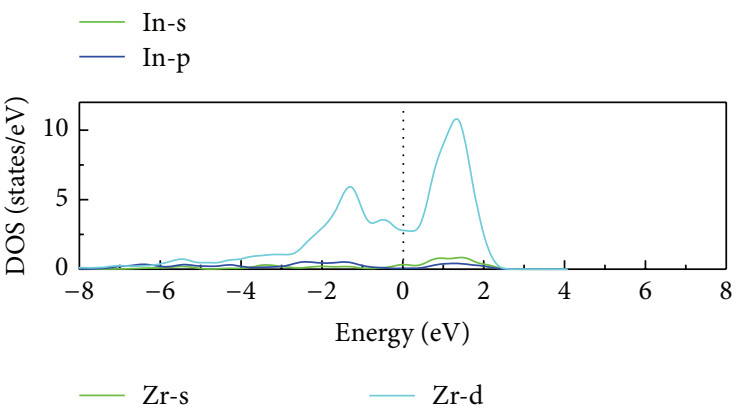

(c) $\mathrm{In}_{2} \mathrm{Zr}$

(d) $\alpha-\mathrm{In}_{3} \mathrm{Zr}$

FIGURE 3: Total and partial electronic densities of states near Fermi level of In-Zr intermetallics. The dotted lines denote the Fermi level $E_{F}$. 
for InZr among these binary In-Zr phases, implying that it possesses the strongest metallicity and hence it has the best ductility. This result has a well consistency with the ductility estimated from the $G / K$ and Poisson's ratio values.

Furthermore, we can estimate the structural stability of intermetallic compounds through different density of states at the Fermi levels [16]. Usually, the lower the value at Fermi levels, the more strong the stability of the structure. From Figure 3, InZr has the minimum value of density of states and in consequence has the most stable structure, which has a great consistency to our results of formation enthalpy.

3.4. Thermodynamic Properties. Debye temperature was calculated to provide insight into the thermodynamics behaviors. The values of Young's modulus, bulk modulus, and shear modulus were obtained from the above section and then put into the following formula [16]:

$$
\begin{aligned}
\Theta_{D} & =\frac{h}{k}\left[\frac{3 n}{4 \pi}\left(\frac{N_{A} \rho}{M}\right)\right]^{1 / 3} v_{m} \\
v_{m} & =\left[\frac{1}{3}\left(\frac{2}{v_{s}^{3}}+\frac{1}{v_{l}^{3}}\right)\right]^{-1 / 3}, \\
v_{l} & =\sqrt{\frac{B+(4 / 3) G}{\rho}} \\
v_{s} & =\sqrt{\frac{G}{\rho}}
\end{aligned}
$$

where $\Theta_{D}$ is Debye temperature, $h$ is Planck's constant, $k$ is Boltzmann's constant, $n$ is the number of atoms per formula, $M$ is the molecular weight, $v_{m}, v_{l}$, and $v_{s}$ are the average wave velocity, the longitudinal, and shear velocity, respectively, and $\rho$ is the theoretical density of the compound. $B$ and $G$ are the bulk modulus and shear modulus, respectively. The values of Debye temperature, average wave velocity, longitudinal, and shear wave velocity for each compound are listed in Table 5. As we all know the strength of chemical bonding can be characterized by Debye temperature which is inverse to the molecular weight $[16,23]$. Stronger chemical bonding favors higher Debye temperature. As shown in Table 5, we can find that the highest $\Theta_{D}$ value is $314.9 \mathrm{~K}$ for $\mathrm{InZr}_{3}$, and the lowest one is ascribed to $\alpha-\mathrm{In}_{3} \mathrm{Zr}$. Therefore, we can conclude that the covalent bonds in $\mathrm{InZr}_{3}$ are stronger than the others.

\section{Conclusions}

In summary, we have performed first-principle calculations to investigate the structural, mechanical, electronic, and thermodynamic properties of In-Zr compounds with different indium concentrations. The calculated results show that the lattice parameters of all compounds are in good agreement with the obtained experimental data and theoretical results. The calculated formation enthalpy indicates that In $\mathrm{Zr}$ is more stable than the others.
TABLE 5: Sound velocity, density, and Debye temperature of In-Zr compounds.

\begin{tabular}{lccccc}
\hline Phase & Density $\left(\mathrm{g} / \mathrm{cm}^{3}\right)$ & $v_{s}$ & $v_{l}$ & $v_{m}$ & $\Theta_{D}$ \\
\hline $\mathrm{InZr}_{3}$ & 7.201 & 2676.875 & 4800.011 & 2980.477 & 314.897 \\
$\mathrm{InZr}$ & 7.662 & 2476.89 & 4504.19 & 2760.842 & 291.892 \\
$\mathrm{In}_{2} \mathrm{Zr}$ & 7.747 & 2467.895 & 4400.46 & 2746.552 & 287.773 \\
$\alpha-\mathrm{In}_{3} \mathrm{Zr}$ & 7.876 & 2453.963 & 4237.448 & 2723.632 & 285.176 \\
\hline
\end{tabular}

The calculated elastic constants show that all the compounds are mechanically stable (Table 3). The bulk modulus and Young's modulus decrease linearly with increasing indium content. The calculated shear modulus and Young's modulus of $\mathrm{InZr}_{3}$ are $51.6 \mathrm{GPa}$ and $131.509 \mathrm{GPa}$, respectively, which are higher than the other compounds. All In-Zr compounds exhibit ductile behavior but $\alpha$ - $\operatorname{In}_{3} \mathrm{Zr}$ exhibits brittle behavior. We conclude that the variation of mechanical properties is related not only to the indium concentration, but also to the bonding state in the In-Zr compounds.

\section{Competing Interests}

The authors declare that they have no competing interests.

\section{Acknowledgments}

This research work is supported by the National Natural Science Foundation of China (51361002 and 51161002), the Program for New Century Excellent Talents in University of China (NCET-12-0650), and the Training Plan of High-Level Talents of Guangxi University (2015).

\section{References}

[1] H. Saitovitch, P. R. J. Silva, J. T. Cavalcante, and M. Forker, "Zirconium-indium intermetallic compounds investigated by measurements of nuclear electric quadrupole interactions," Journal of Alloys and Compounds, vol. 505, no. 1, pp. 157-162, 2010.

[2] H. Okamoto, “The In-Zr (Indium-Zirconium) system," Bulletin of Alloy Phase Diagrams, vol. 11, no. 2, pp. 150-152, 1990.

[3] M. F. Zumdick, G. A. Landrum, R. Dronskowski, R.-D. Hoffmann, and R. Pöttgen, "Structure, chemical bonding, and properties of ZrIn2, IrIn2, and Ti3Rh2In3," Journal of Solid State Chemistry, vol. 150, no. 1, pp. 19-30, 2000.

[4] S. V. Meschel and O. J. Kleppa, "Standard enthalpies of formation of some transition metal indium compounds by high temperature direct synthesis calorimetry," Journal of Alloys and Compounds, vol. 333, no. 1-2, pp. 91-98, 2002.

[5] K. Yamanaka, M. Mori, and A. Chiba, "Developing high strength and ductility in biomedical Co-Cr cast alloys by simultaneous doping with nitrogen and carbon," Acta Biomaterialia, vol. 31, pp. 435-447, 2016.

[6] M. D. Segall, P. J. D. Lindan, M. J. Probert et al., "First-principles simulation: ideas, illustrations and the CASTEP code," Journal of Physics: Condensed Matter, vol. 14, no. 11, pp. 2717-2744, 2002.

[7] D. Vanderbilt, "Soft self-consistent pseudopotentials in a generalized eigenvalue formalism," Physical Review B, vol. 41, no. 11, pp. 7892-7895, 1990. 
[8] J. P. Perdew, K. Burke, and M. Ernzerhof, "Generalized gradient approximation made simple," Physical Review Letters, vol. 77, no. 18 , pp. 3865-3868, 1996.

[9] K. Anderko, "Beitrag zu den binären Systemen des Titans mit Gallium, Indium und Germanium und des Zirkons mit Gallium und Indium," Zeitschrift für Metallkunde, vol. 49, no. 4, pp. 165$172,1958$.

[10] J. C. Uy and A. A. Burr, "The solute metallic valence as an index of phase stabilization in zirconium-based alloys," Transactions of the Metallurgical Society of AIME, vol. 224, no. 2, p. 204, 1962.

[11] E. C. Moshopoulou, R. M. Ibberson, J. L. Sarrao, J. D. Thompson, and Z. Fisk, "Structure of $\mathrm{Ce}_{2} \mathrm{RhIn}_{8}$ : an example of complementary use of high-resolution neutron powder diffraction and reciprocal-space mapping to study complex materials," Acta Crystallographica Section B: Structural Science, vol. 62, no. 2, pp. 173-189, 2006.

[12] B. D. Lichter, "Precision lattice parameter determination of zirconium-oxygen solid solution," Transactions of the Metallurgical Society of AIME, vol. 218, 1960.

[13] Q.-J. Liu, N.-C. Zhang, F.-S. Liu, and Z.-T. Liu, "Structural, mechanical and electronic properties of OsTM and $\mathrm{TMOs}_{2}$ (TM = Ti, Zr and Hf): first-principles calculations," Journal of Alloys and Compounds, vol. 589, pp. 278-282, 2014.

[14] Z.-J. Wu, E.-J. Zhao, H.-P. Xiang, X.-F. Hao, X.-J. Liu, and J. Meng, "Crystal structures and elastic properties of superhard $\mathrm{IrN}_{2}$ and $\mathrm{IrN}_{3}$ from first principles," Physical Review B, vol. 76, no. 5, Article ID 054115, 15 pages, 2007.

[15] Q.-J. Liu, H. Tian, and Z.-T. Liu, "Mechanical properties and electronic structures of the Hf-Si system: first-principles calculations," Solid State Communications, vol. 205, pp. 39-45, 2015.

[16] Z. Zhou, X. Zhou, and K. Zhang, "Phase stability, electronic structure and mechanical properties of $\operatorname{IrBx}(x=0.9,1.1)$ : firstprinciples calculations," Computational Materials Science, vol. 113, pp. 98-103, 2016.

[17] L.-H. Li, W.-L. Wang, L. Hu, and B.-B. Wei, "First-principle calculations of structural, elastic and thermodynamic properties of Fe-B compounds," Intermetallics, vol. 46, pp. 211-221, 2014.

[18] A. Kumar, A. Chernatynskiy, M. Hong, S. R. Phillpot, and S. B. Sinnott, "An ab initio investigation of the effect of alloying elements on the elastic properties and magnetic behavior of $\mathrm{Ni}_{3} \mathrm{Al}$," Computational Materials Science, vol. 101, pp. 39-46, 2015.

[19] Y. Pan, M. Wen, L. Wang, X. Wang, Y. H. Lin, and W. M. Guan, "Iridium concentration driving the mechanical properties of iridium-aluminum compounds," Journal of Alloys and Compounds, vol. 648, Article ID 34748, pp. 771-777, 2015.

[20] B. Huang, Y.-H. Duan, W.-C. Hu, Y. Sun, and S. Chen, "Structural, anisotropic elastic and thermal properties of $\mathrm{MB}$ ( $\mathrm{M}=\mathrm{Ti}, \mathrm{Zr}$ and $\mathrm{Hf}$ ) monoborides," Ceramics International, vol. 41, no. 5, pp. 6831-6843, 2015.

[21] V. V. Bannikov, I. R. Shein, and A. L. Ivanovskii, "Structural, elastic, electronic properties and stability trends of 1111-like silicide arsenides and germanide arsenides MCuXAs $(M=\mathrm{Ti}$, Zr, Hf; $X=\mathrm{Si}, \mathrm{Ge}$ ) from first principles," Journal of Alloys and Compounds, vol. 533, pp. 71-78, 2012.

[22] H. Hu, X. Wu, R. Wang, W. Li, and Q. Liu, "Phase stability, mechanical properties and electronic structure of TiAl alloying with W, Mo, Sc and Yb: first-principles study," Journal of Alloys and Compounds, vol. 658, pp. 689-696, 2016.

[23] G. Yi, X. Zhang, J. Qin et al., "Mechanical, electronic and thermal properties of $\mathrm{Cu}_{5} \mathrm{Zr}$ and $\mathrm{Cu}_{5} \mathrm{Hf}$ by first-principles calculations," Journal of Alloys and Compounds, vol. 640, pp. 455-461, 2015.

[24] E. Jain, G. Pagare, S. S. Chouhan, and S. P. Sanyal, "Electronic structure, phase stability and elastic properties of ruthenium based four intermetallic compounds: Ab-initio study," Intermetallics, vol. 54, pp. 79-85, 2014.

[25] S. Liu, Y. Zhan, J. Wu, and X. Wei, "Insight into structural, mechanical, electronic and thermodynamic properties of intermetallic phases in $\mathrm{Zr}$-Sn system from first-principles calculations," Journal of Physics and Chemistry of Solids, vol. 86, pp. 177-185, 2015.

[26] Y. Zhao, H. Hou, Y. Zhao, and P. Han, "First-principles study of the nickel-silicon binary compounds under pressure," Journal of Alloys and Compounds, vol. 640, pp. 233-239, 2015.

[27] Y. Pan, Y. Lin, X. Wang et al., "Structural stability and mechanical properties of $\mathrm{Pt}-\mathrm{Zr}$ alloys from first-principles," Journal of Alloys and Compounds, vol. 643, pp. 49-55, 2015.

[28] A. Lee, G. Etherington, and C. N. J. Wagner, "Partial structure functions of amorphous $\mathrm{Ni}_{35} \mathrm{Zr}_{65}$," Journal of Non-Crystalline Solids, vol. 61-62, no. 1, pp. 349-354, 1984.

[29] A. W. Weeber and H. Bakker, "Extension of the glass-forming range of $\mathrm{Ni}-\mathrm{Zr}$ by mechanical alloying," Journal of Physics F: Metal Physics, vol. 18, no. 7, pp. 1359-1369, 1988.

[30] J. Du, B. Wen, R. Melnik, and Y. Kawazoe, "First-principles studies on structural, mechanical, thermodynamic and electronic properties of $\mathrm{Ni}-\mathrm{Zr}$ intermetallic compounds," Intermetallics, vol. 54, pp. 110-119, 2014. 

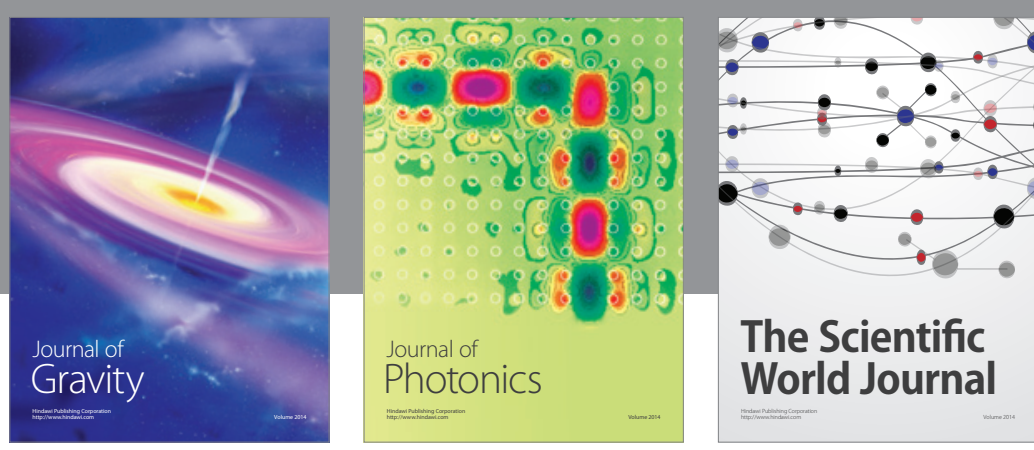

The Scientific World Journal
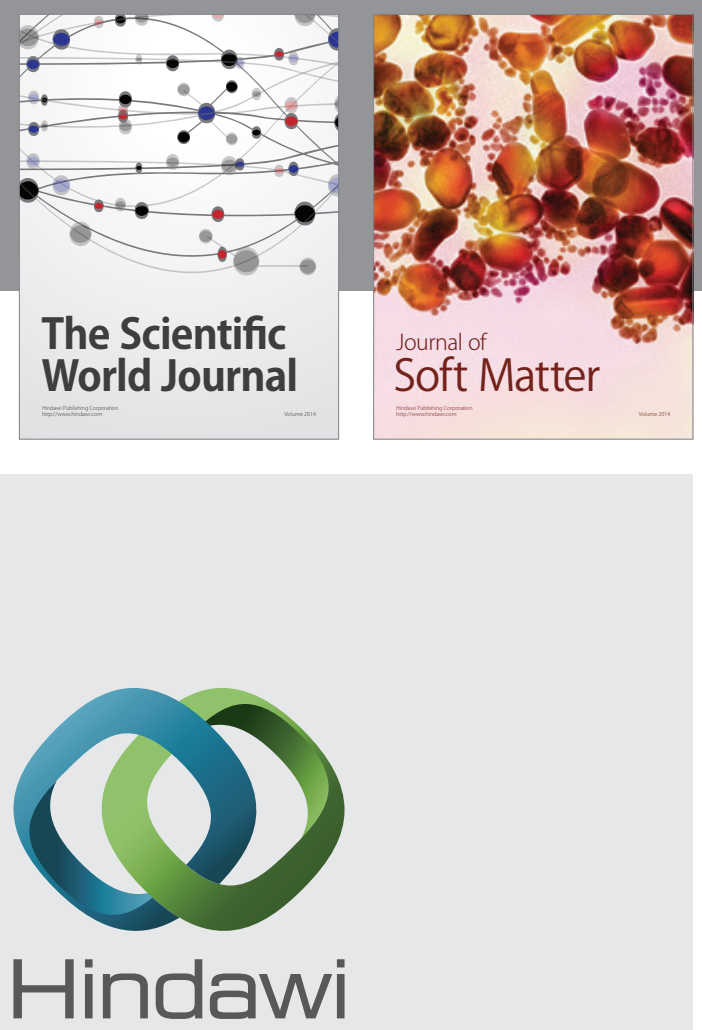

Submit your manuscripts at

http://www.hindawi.com

nternational Journal of

Statistical Mechanics
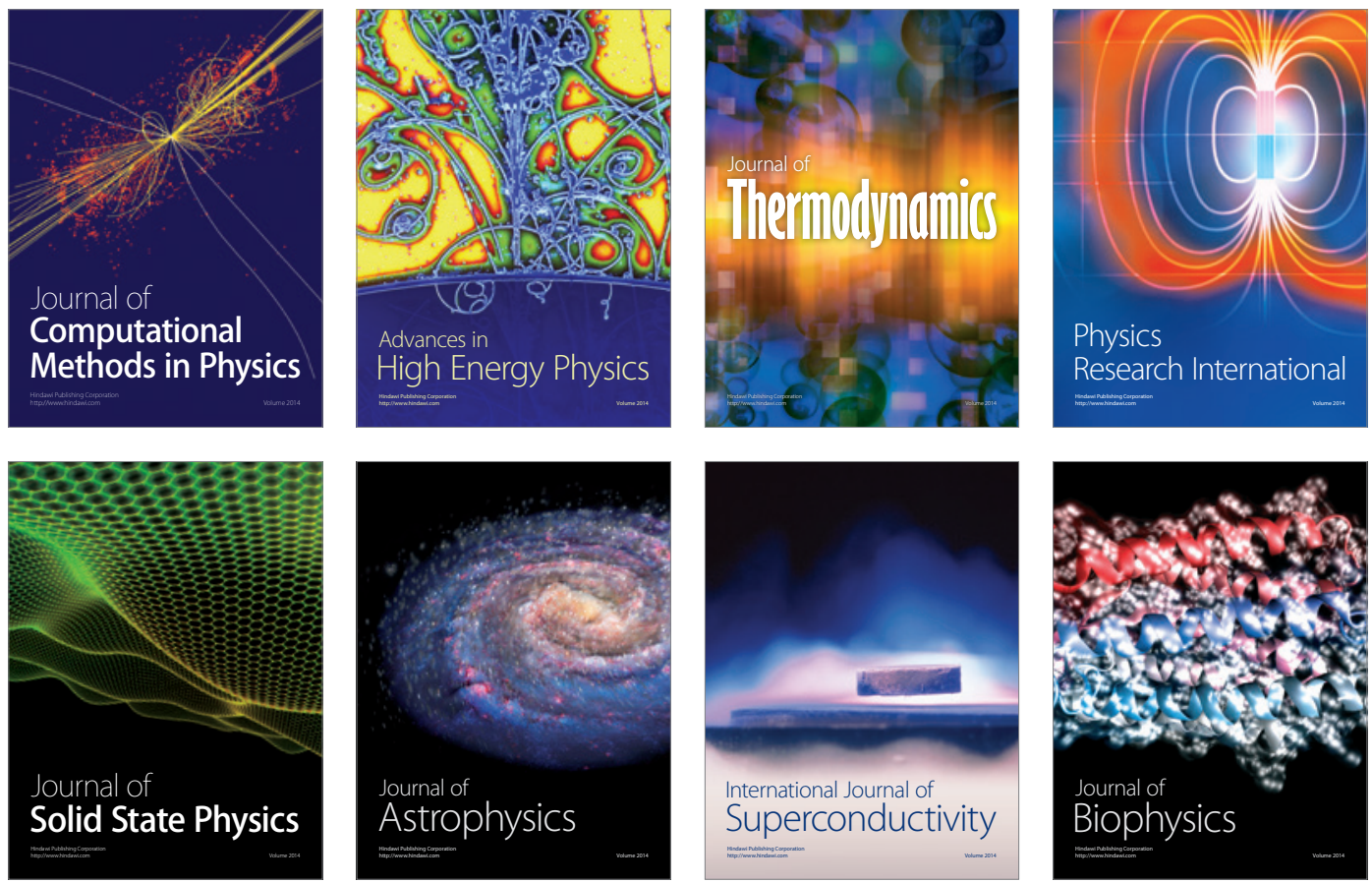
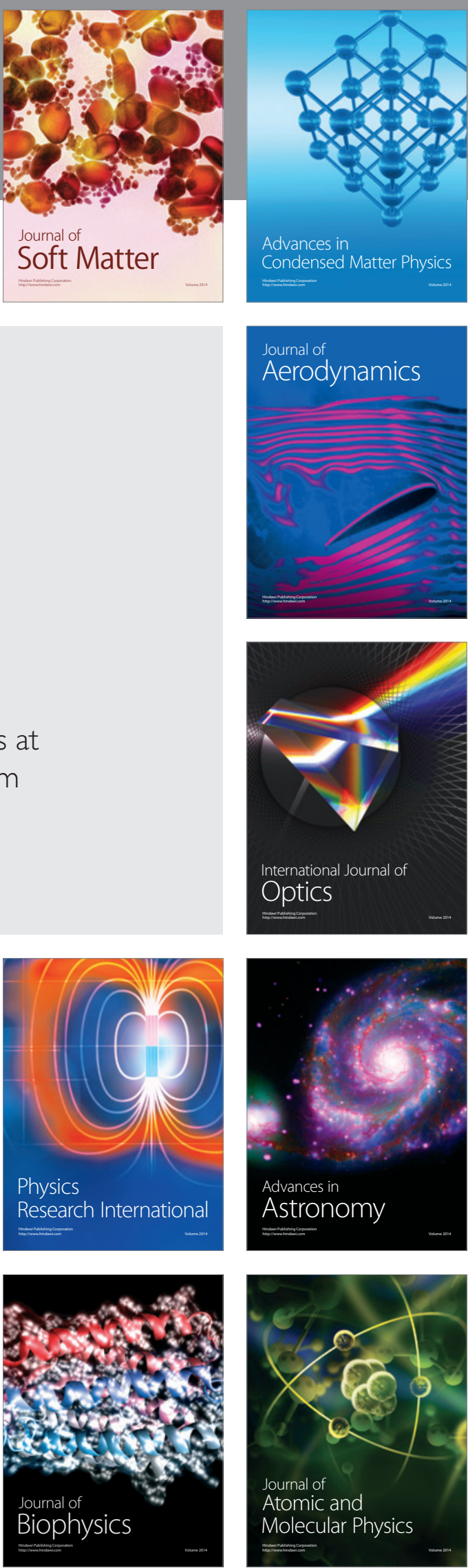